\title{
Malaria Outbreak Investigation in Siansundu, Binga District, Matabeleland North Province, Zimbabwe, 2013
}

\author{
Cynthia Ncube $^{1 *}$, Chiedza Sibanda ${ }^{2}$, Nyasha Masuka $^{3}$ \\ ${ }^{1}$ Department of Community Medicine, University of Zimbabwe, Harare, Zimbabwe \\ ${ }^{2}$ City Health Department, Bulawayo City Council, Bulawayo, Zimbabwe \\ ${ }^{3}$ Ministry of Health and Child Care, Matabeleland North Provincial Medical Directorate, \\ Zimbabwe
}

\begin{abstract}
Malaria is of public health importance in Zimbabwe. A sharp rise in the number of malaria cases in Binga district was noted during week five in 2013. On further analysis, the Siansundu clinic was found to be in an outbreak situation. The study was conducted to determine factors associated with contracting malaria in Binga District, Matabeleland North, Zimbabwe. An unmatched 1: 1 casecontrol study was conducted in Siansundu, Binga, among 124 residents. A case was a person who presented with malaria symptoms, a control was a person who was a neighbour of a case and did not suffer from typical malaria symptoms or had a negative RDT result from the $1^{\text {st }}$ of January 2013. A pre-tested interviewer-administered questionnaire and a checklist were used to collect data. Data were analyzed using Epi Info, where odds ratios and $p$ values were calculated. Risk factors for contracting malaria were: Fetching water at night (OR 2.55, p-value 0.04); Having inadequate mosquito nets per sleeping space (OR 3.596, p-value 0.036); Worshipping and praying outside at night (OR 3.417, p-value 0.0006). Wearing long clothing at night was a protective factor against contracting malaria (OR 0.156, p-value 0.001). The case fatality rate was $0.43 \%$. The district was not prepared for the outbreak and responded late. Educational strategies to address the risk factors for malaria and regular meetings by the emergency preparedness and response team were recommended to prevent future outbreaks and aid outbreak preparedness.
\end{abstract}

Keywords: Binga, Case, Control, Malaria outbreak, Risk Factors.

\section{Introduction}

Malaria is an acute febrile illness caused by a parasite of the genus Plasmodium. The malaria parasite is transmitted by female Anopheles mosquitoes, which bite mainly between dusk and dawn [1]. The incubation period for malaria is 7 to 30 days. Variable clinical features of malaria include fever, chills, headache, muscular aching and weakness, vomiting, cough, diarrhoea, abdominal pain, and general body malaise [2, 3]. Early diagnosis and treatment of malaria reduces disease, prevents deaths, and contributes to reducing malaria transmission [4].
Malaria is a global public health problem, with approximately half of the world's population being at risk of malaria. Children account for $85 \%$ of all malaria deaths globally. According to the World Health Organisation, there were about 219 million cases of malaria in 2010 globally and an estimated 660000 deaths [1]. There are 900000 deaths per year in Africa, mainly in children less than 5 years of age and pregnant women [5]. In Africa, a child dies every minute from malaria. The African Region accounts for $85 \%$ of malaria cases and $90 \%$ of malaria deaths worldwide [6, 7].

In Zimbabwe, malaria continues to be a major public health problem. Over half of the 
population of 13 million is at risk of contracting malaria. It is the third common cause of morbidity and mortality in the country, after HIV and AIDS and Tuberculosis across all age groups. Annually close to 1.5 million malaria episodes are reported whilst an average of 1000 people die from this disease. Malaria accounts for $30 \%$ of outpatients at clinics and $40 \%$ of hospital admissions. Various strategies have been put in place to prevent and control malaria. Case management is one of the extremely important strategies alongside such strategies as integrated vector control; prevention of malaria in pregnancy; information education communication and advocacy; epidemic preparedness and response; monitoring and evaluation as well as operational research [8].

Matabeleland North Province is one of the provinces in the country with a high burden of malaria. The province has a total population of approximately 780000 of which 541425 are at risk of contracting malaria. Although all the seven districts in the province are affected, Binga, Hwange, and Lupane have the greatest malaria burden in the province. The province had a total of 28 malaria deaths in 2010-2011, and the majority of these deaths occurred in Binga District, which is prone to malaria outbreaks. A malaria outbreak was last reported in the district (Siansundu) in 2010 [9].

A sharp rise in the number of malaria cases in Binga district was noted during week five. On further analysis by the health centre, Siansundu clinic was found to be in an outbreak situation since week five [10]. It was against this background that an investigation was carried out in order to assist in controlling the outbreak and make appropriate recommendations.

The investigation sought to find the factors associated with contracting malaria in the Siansundu area, Binga. The first null hypothesis was that there is no association between sitting outside late at night and contracting malaria. The second null hypothesis was that there is no association between sleeping under a treated mosquito net daily and contracting malaria. The broad objective was to establish the factors associated with the malaria outbreak in Siansundu, Binga. The specific objectives were to; characterize the malaria outbreak by person, place, and time; to identify the risk factors associated with contracting malaria; to assess community knowledge on malaria; to evaluate malaria case management; to assess the outbreak preparedness and response by Binga District.

\section{Methods}

An unmatched 1:1 case-control study was conducted in Siansundu, Binga District. A case was a person residing in Siansundu who presented with typical malaria symptoms (headache, chills, fever, joint pains, vomiting, general body malaise, abdominal pains), who had a positive Rapid Diagnostic Test (RDT) result from the 1st of January 2013 to the last day of the study. A control was a person residing in Siansundu who was a neighbour of a case and did not suffer from typical malaria symptoms or had a negative RDT result from the $1^{\text {st }}$ of January 2013 to the last day of the study. The study population comprised of all people who had been residing in the Siansundu area, Binga District, for at least six months prior to the outbreak.

A minimum sample size of 120 was calculated; 60 cases and 60 controls. The line list of cases at Siansundu Clinic was used as the sampling frame for cases. Study participants were systematically selected from the line list. Controls were randomly selected from the neighbours of the cases using the lottery method.

All cases who; had been resident in Siansundu for at least 6 months; were listed on the line list of cases at Siansundu Clinic and had a positive RDT result were sampled into the study. Cases who; had not been resident in Siansundu for at least 6 months; did not have a positive RDT result; were too ill or too old such 
that their ability to recall past exposures was impaired were excluded from the study.

Interviewer-administered questionnaires were used to collect information from cases, controls, and health workers. A review of patient records was done to assess the appropriateness of case management. A checklist was used to assess the timeliness and quality of the outbreak preparedness, detection, investigation, and response. An environmental assessment was done in the homes of cases and controls to assess for cleanliness around the homestead, presence of water bodies near the homestead, presence of long grass or empty tins around the homestead, and the presence of breeding mosquitoes.

Permission to conduct the study was sought from the Provincial Medical Director (PMD) for Matabeleland North, the District Medical Officer (DMO) for Binga District, the Health Studies Office (HSO), and the study participants.

Data was entered into and analyzed using Epi info 3.5.3 to generate frequencies, odds ratios, and their $95 \%$ confidence intervals. A set of 15 knowledge questions were used to score knowledge where 0-5 correct responses meant poor knowledge, 6-10 correct responses meant fair knowledge, and 11-15 correct responses represented good knowledge on malaria. Univariate and bivariate analyses were done to check for associations between variables. Significant differences between cases and controls in terms of exposure to risk factors were tested for using the Chi-square test. $\mathrm{P}$ values less than 0.05 were considered statistically significant. Confounding was assessed using stratified analysis.

\section{Results}

A total of one hundred and twenty-four (124) respondents were enrolled into the study, sixtytwo cases and sixty-two controls. Thirty of the cases were male and thirty-two females. Thirty of the controls were females and thirty-two males. Cases and controls were comparable as there were no statistically significant differences in their demographic profiles. This is shown in Table 1.

Table1. Demographic Characteristics of Cases and Controls, Siansundu, Binga, 2013

\begin{tabular}{|c|c|c|c|}
\hline Variable & Cases $\mathrm{N}=62(\%)$ & Controls $\mathrm{N}=62(\%)$ & $P$ value \\
\hline \multicolumn{4}{|l|}{ Sex } \\
\hline Female & $32(52)$ & $30(48)$ & \multirow[t]{2}{*}{0.857} \\
\hline Male & $30(48)$ & $32(52)$ & \\
\hline \multicolumn{4}{|l|}{ Marital Status } \\
\hline Married & $18(29)$ & $19(31)$ & \multirow[t]{4}{*}{0.7491} \\
\hline Single & $8(13)$ & $9(14)$ & \\
\hline Widowed & $0(0)$ & $1(2)$ & \\
\hline Child Under 16 & $36(58)$ & $33(53)$ & \\
\hline \multicolumn{4}{|l|}{ Educational Level } \\
\hline None & $6(10)$ & $6(10)$ & \multirow[t]{4}{*}{0.794} \\
\hline Primary & $39(63)$ & $38(61)$ & \\
\hline Secondary & $17(27)$ & $18(29)$ & \\
\hline Tertiary & 0 & 0 & \\
\hline \multicolumn{4}{|l|}{ Occupation } \\
\hline Employed & $4(6)$ & $4(5)$ & \multirow[t]{4}{*}{0.9986} \\
\hline Unemployed & $21(35)$ & $22(35)$ & \\
\hline Student & $28(46)$ & $31(50)$ & \\
\hline Minor & & $6(10)$ & \\
\hline
\end{tabular}




\begin{tabular}{|l|l|l|l|}
\hline Religion & $8(13)$ & $29(47)$ & \multirow{2}{*}{0.8221} \\
\hline Pentecostal & $22(36)$ & $6(10)$ & \\
\hline Protestant & $2(3)$ & $12(19)$ & \\
\hline Apostolic & $26(42)$ & $6(10)$ & \\
\hline Traditionalist & $2(3)$ & $9(14)$ & \\
\cline { 1 - 2 } None & $14(7: 22)$ & $13.5(8: 23)$ & \\
\cline { 1 - 3 } Median age (Q1:Q3) & &
\end{tabular}

The first outbreak case was seen at the clinic

Provincial team investigated on the $28^{\text {th }}$ of during week five. The clinic staff detected the outbreak on the $4^{\text {th }}$ of February and notified the district on the same day. The district investigated on the $14^{\text {th }}$ of February 2013. The

February 2013 and also brought larviciding chemicals during the visit. The number of cases kept increasing gradually, decreased markedly in week 10, and peaked in week 15- Figure 1.

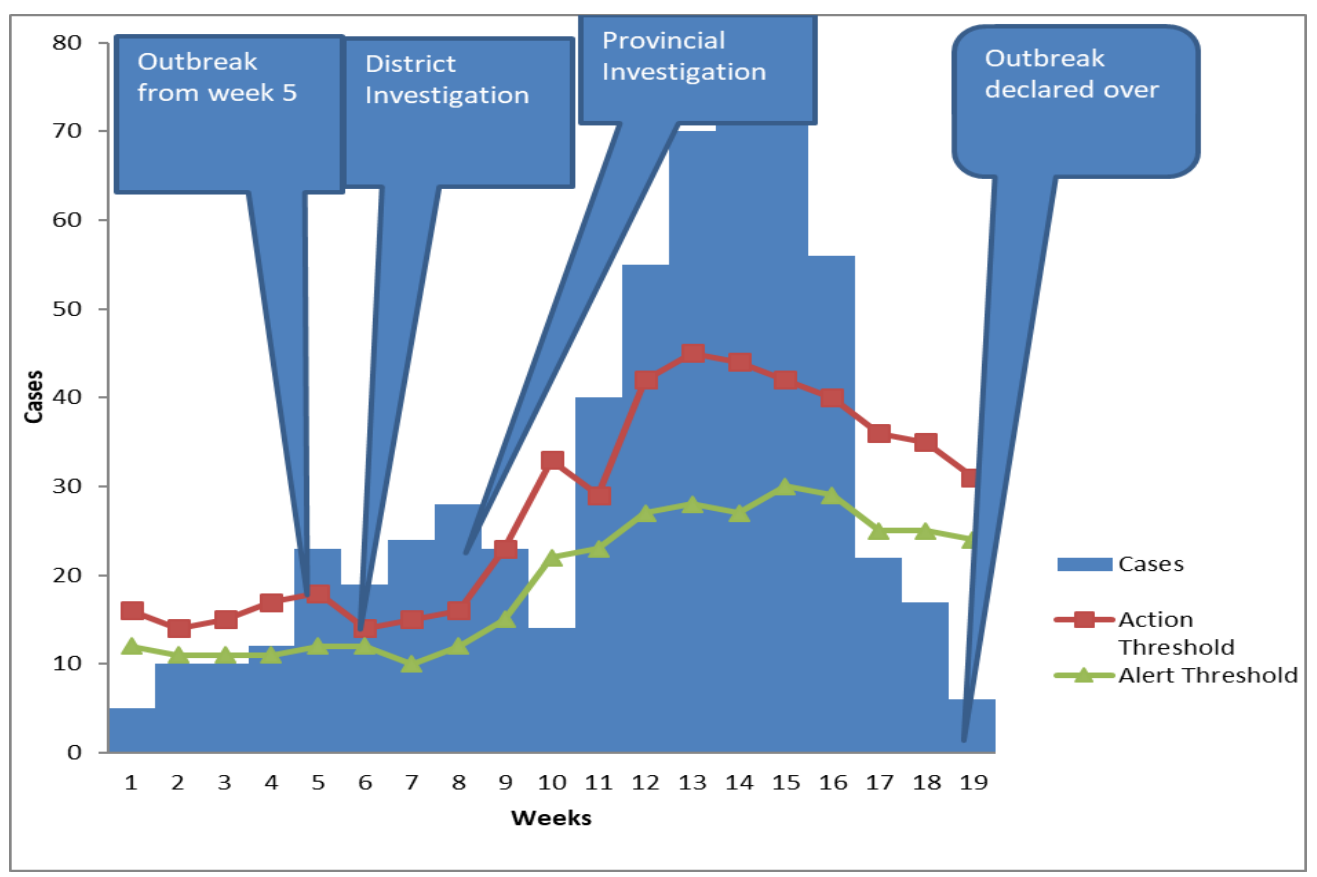

Figure 1. Epicurve-Malaria Outbreak in Siansundu, Binga, 2013

Larviciding, community health education, distribution of Coartemether to VHWs, and scooping of larvae were part of the measures which were done in an effort to control the outbreak. An outreach clinic was held for three days and then stopped due to a shortage of fuel. The outbreak was declared over in week nineteen.

A total of two hundred and thirty-three cases were line listed at the clinic. The most affected age group was 5-10 years which contributed
$23 \%$ (14) cases, and the least affected age group was $26-30$ years with only $7 \%$ (4) cases. Fortythree $(69 \%)$ of the cases reported having ever heard about malaria compared with $50(81 \%)$ of the controls. The mean knowledge score for cases and controls was 3.75(SD 3.05) and 4.48(SD 3.05), respectively. The difference in knowledge levels between cases and controls was not statistically significant, as shown by Table 2. 
Table 2. Knowledge Score of Malaria among Cases and Controls, Siansundu, 2013

\begin{tabular}{|l|l|l|l|}
\hline \multirow{2}{*}{ Knowledge Score } & Cases & Controls & \multirow{2}{*}{ P Value } \\
\cline { 2 - 3 } & $\mathbf{N = 6 2}(\boldsymbol{\%})$ & $\mathbf{N}=\mathbf{6 2}(\boldsymbol{\%})$ & \\
\hline Poor Knowledge & $41(68.4)$ & $39(62.9)$ & \multirow{2}{*}{0.617} \\
\hline Fair Knowledge & $19(30.6)$ & $20(32.3)$ & \\
\hline Good Knowledge & $2(3.2)$ & $3(4.8)$ & \\
\hline
\end{tabular}

Most of the cases (40\%) presented at the clinic with a headache. The other major presenting symptoms were fever, chills, and general body malaise. Fifty-eight (93.5) \%) of the cases did not seek treatment anywhere else before presenting at the clinic. The five (8\%) who did first consult the village health worker. None of the five were given anti-malarial treatment as none of the Community Based Workers had the drugs. The majority $(87 \%)$ of the cases had uncomplicated malaria and were treated with Coartemether. Forty-nine (77\%) of the cases were also given paracetamol in addition to Coartemether. Only nine (14, 5\%) cases were treated with quinine. There was one death of under-five females recorded during week nine, which translates to a case fatality of $0.43 \%$ up to week thirteen.

The clinic had a complement of six members of staff, and four were interviewed. All four were trained on malaria case management, but none were trained in Integrated Disease Surveillance and Response (IDSR). Four health workers were interviewed at the district hospital. Only one out of the four was not trained in case management. Only two out of the four were trained in IDSR.

Painting or washing walls after spraying were found to be a risk factor for contracting malaria (OR 2.54.p value 0.181). However, this relationship was not statistically significant. Similarly, having long grass around the homestead was also found to be a risk factor for contracting malaria, although this was not statistically significant (OR 1.98, p-value 0.150). Fetching water at night or before sunrise was found to be a significant risk factor for contracting malaria (OR 2.55, p-value 0.04).

Having inadequate mosquito nets per sleeping space was found to be a risk factor for contracting malaria (OR 3.596, p-value 0.036). Worshipping and praying outside at night which is typical of the apostolic sect members, was found to be significantly associated with contracting malaria (OR 3.417, p-value 0.0006). Wearing long clothing at night was found to be a significant protective factor against contracting malaria in Siansundu (OR 0.156, pvalue 0.001$)$. The risk factors are shown in Table 3.

Table 3. Risk Factors for Contracting Malaria, Siansundu, Binga, 2013

\begin{tabular}{|l|l|l|l|l|}
\hline \multirow{2}{*}{ Malaria Risk Factor } & Cases & Controls & OR & \multirow{2}{*}{ P-value } \\
\cline { 2 - 4 } & $\mathbf{N = 6 2 ( \% )}$ & $\mathbf{N = 6 2}(\mathbf{\%})$ & & \\
\hline Burning mosquito coil at night & $3(5)$ & $2(3)$ & 1.525 & 0.50 \\
\hline Painting/washing wall after spraying & $10(16)$ & $5(8)$ & 2.54 & 0.181 \\
\hline Homestead near water body & $41(66)$ & $37(60)$ & 1.32 & 0.577 \\
\hline Long grass around homestead & $50(81)$ & $42(68)$ & 1.98 & 0.150 \\
\hline Fetching water before sunrise & $18(29)$ & $11(18)$ & 6.4 & $0.04^{*}$ \\
\hline Inadequate nets in household & $46(74)$ & $33(53)$ & 3.596 & $0.036^{*}$ \\
\hline Worshipping outside at night & $26(42)$ & $12(19)$ & 3.417 & $0.006^{*}$ \\
\hline Protective Factors & & & & \\
\hline Wearing long clothing at night & $4(6)$ & $19(31)$ & 0.156 & $0.001^{*}$ \\
\hline House sprayed & $47(76)$ & $52(84)$ & 0.602 & 0.370 \\
\hline
\end{tabular}




\begin{tabular}{|l|l|l|l|l|}
\hline All rooms sprayed & $43(69)$ & $48(77)$ & 0.670 & 0.454 \\
\hline Ever heard of malaria & $43(69)$ & $50(81)$ & 0.54 & 0.213 \\
\hline Empty containers near homestead & $9(15)$ & $10(16)$ & 0.880 & 1.000 \\
\hline Ever received malaria HE & $22(35)$ & $27(44)$ & 0.852 & 0.857 \\
\hline Sleeping under LLIN & $36(61)$ & $41(66)$ & 0.810 & 0.709 \\
\hline Sitting indoors at night & $10(16)$ & $11(18)$ & 0.892 & 1.000 \\
\hline
\end{tabular}

*P-value is less than 0.05 and statistically significant.

On stratified analysis, confounding was not present. Fetching water at night was modified by gender, such that females who fetched water at night were 1.64 times more likely to contract malaria compared to females who did not. Similarly, males who fetched water at night were 6.4 times more likely to contract malaria compared to those males who did not. Worshipping outside at night was modified by gender such that females who worshipped outside at night were 2.55 times more likely to contract malaria. Similarly, males who worshiped outside at night were 4.94 times more likely to contract malaria than those who did not.

The inadequacy of nets per household was modified by gender. Females who belonged to households with inadequate nets were 1.8 times more likely to contract malaria than those who belonged to households with adequate nets. Similarly, males who belonged to households with inadequate nets were 3.59 times more likely to contract malaria compared to those males who belonged to households with adequate nets.

It was reported that hard-to-reach areas did not fully benefit from net registration and distribution. This was due to a short registration period and few distribution points. The indoor residual spraying programme coverages for 2011 and 2012 were all above 90\%. However, hard-to-reach areas were left unsprayed due to a limited number of days, communication problems, and water shortages.

Only thirteen of the twenty permanent and seasonal established water bodies in Siansundu had been treated at the time of the investigation. The treatment of these water bodies commenced during the rainy season, affecting the chemical function of Bug Stop. In addition, distances between the water bodies in Lubanda and Saba ward are too long, causing a strain in checking chemical functioning and possible reduction of larvae. The environmental assessment results are shown in Table 4.

Table 4. Environmental Assessment, Siansundu, Binga, 2013

\begin{tabular}{|l|l|l|l|}
\hline \multirow{2}{*}{ Variable } & Cases & Controls & \multirow{2}{*}{ P - value } \\
\cline { 2 - 3 } & $\mathbf{N = 6 2 ( \% )}$ & $\mathbf{N = 6 2 ( \% )}$ & \\
\hline House not sprayed & $14(23)$ & $10(16.1)$ & 0.467 \\
\hline Washed walls after spraying & $10(16)$ & $5(8)$ & 0.181 \\
\hline Lived near water body & $41(66)$ & $37(60)$ & 0.577 \\
\hline Had long grass around the homestead & $50(81)$ & $42(68)$ & 0.150 \\
\hline Had empty containers around homestead & $9(14.5)$ & $10(16.1)$ & 1.000 \\
\hline
\end{tabular}

The interval between the day the clinic notified the district and the date of district field investigation was nine (9) days. The interval between the notification of the outbreak to the district and the concrete response by the district was twenty-seven days. The corresponding period for the province was nine days when the province brought larviciding chemicals to the clinic.

Adequate drugs and supplies were available at the onset of the outbreak, but the district pharmacy did not have enough RDTs. The 
clinic reported that they usually have very little anti-malarial drugs, which are less than the calculated minimum stock. This was also noted during the investigation.

As part of epidemic preparedness, the district epidemic committee was not meeting regularly. Feedback about the district investigation findings was not given to the health facility or the community. There was no completed line list at the clinic. Malaria thresholds were available. There were no malaria Information, Education, and Communication (IEC) materials available at the clinic, and no case definitions for malaria were seen. A number of recommendations were made in Siansundu [11]. These had only been partially implemented at the time of the study. This information is shown in Table 5.

Table 5. Outbreak Detection, Preparedness and Response, Siansundu, 2013

\begin{tabular}{|l|l|l|l|}
\hline Indicator & Date 1 & Date 2 & Interval \\
\hline $\begin{array}{l}\text { Interval between date action threshold was reached at } \\
\text { the clinic and reporting to the district health team. Target } \\
(<24 \text { hours })\end{array}$ & $4 / 02 / 2013$ & $4 / 04 / 2013$ & $<24$ hours \\
\hline $\begin{array}{l}\text { Interval between notification of district and date of } \\
\text { district field investigation. Target: Within 48hrs }\end{array}$ & $04 / 02 / 2013$ & $14 / 02 / 2013$ & 9 days \\
\hline $\begin{array}{l}\text { Interval between notification of outbreak to district and } \\
\text { concrete response by the district. Target: Within 48 hrs. } \\
\text { of notification }\end{array}$ & $04 / 02 / 2013$ & $31 / 02 / 2013$ & 27 days \\
\hline Indicator & Yes & No & \\
\hline Was a line list completed at the health center? & & No & \\
\hline $\begin{array}{l}\text { Were adequate drugs and supplies available at the onset } \\
\text { of the outbreak? }\end{array}$ & No & \\
\hline
\end{tabular}

\section{Discussion}

More than $60 \%$ of both cases and controls had poor knowledge of malaria. This finding may explain why Siansundu residents continue contracting malaria despite previous outbreaks. The finding is consistent with a study done in Bangladesh where the respondents lacked comprehensive knowledge on different aspects of malaria, which was influenced by the level of poverty and education. The study also found that delay in seeking care was common among these respondents [12]. However, the finding of poor knowledge levels is contrary to findings made by Kinung'hi etal in 2010, where ninety two percent of Tanzanian respondents knew that malaria is transmitted through an infected mosquito bite.

Thirty-five (56.4\%), of the cases presented at the clinic between three and fourteen days, against a national target of two days. The late treatment-seeking behaviour exhibited by the cases is consistent with the findings [11]. Long distances to be covered by the community to get to the health centre and inadequate drug stock by community health workers could be contributory factors. The district was inadequately prepared for the outbreak leading to a shortage of resources. This negatively affected outbreak control and resulted in the outbreak taking longer to contain. This is consistent with the findings of Laiez in Ethiopia, who found out that during the malaria epidemic in 2003, the impact of rapid antimalaria distribution to villages was diminished by delays in delivering the drugs, the late availability of operational funds, and inadequate protocols for early response [13].

None of the cases first sought treatment from traditional or faith healers. This shows that the community of Binga now has faith in the health system, and the clinic is their first port of call when they fall sick. Although late treatmentseeking behaviour is still a pertinent issue, this 
is a step in the right direction towards the provision of health for all by the year 2015 .

Almost $100 \%$ of the health workers interviewed were trained in case management. The nurses had the knowledge on the first line, second-line treatment for uncomplicated malaria as well as treatment of complicated malaria. When health workers are trained in case management, they tend to give proper treatment to patients, resulting in more positive health outcomes. The proper and effective case management given by the nurses at Siansundu clinic resulted in a very low case fatality rate of $0.43 \%$, despite the fact that most patients presented late. However, lack of training in IDSR compromises the effectiveness of health workers in instituting disease control measures.

Fetching water before sunrise was found to be a significant risk factor for contracting malaria in Siansundu. This is biologically plausible since the Anopheles mosquitoes known to transmit malaria bite between sunset and sunrise. This also suggests the presence of infected mosquitoes in large numbers in Siansundu. The findings were consistent with those made in 2010 in Siansundu [11]. Having inadequate nets was found to be a significant risk factor for malaria. This is also biologically plausible because if one has a mosquito net and they sleep under it daily, this reduces their chances of being bitten by the mosquito and consequently contracting malaria. Although net usage still needs to be assessed further, the finding suggests that in Siansundu, those people who have the nets actually use them for the purpose of preventing mosquito bites. The provision of mosquito nets to all who need them might greatly lower the incidence of malaria in Siansundu.

It was also found during the investigation that the net registration period for 2012 was too short, distribution points too few, and that most polygamous families did not get enough nets in Siansundu. An insufficient number of LLINs distributed is one of the barriers to malaria prevention [14]. The shortage of nets could have contributed to the outbreak.

Worshipping outside at night was found to be a significant risk factor for malaria. This behaviour is typical of apostolic sect members, who spend most of their time praying and worshipping up the mountains, especially at night. The risk of contracting malaria in this group was found to be greater for men compared to women, probably due to the fact that men spend more time on the mountains due to their leading role in the church. Wearing long clothing at night was found to be protective against malaria.

IRS had its own challenges in 2012. The presence of spray operators from other provinces meant that communication with community members was a problem. As a result, some spray operators could not locate their targeted spraying areas (got lost), could not give clear instructions to the community about the provision of water for mixing the chemical and could not communicate clearly that community members were not supposed to wash or redecorate walls after spraying. Nonspraying of houses could have contributed to the outbreak. Similar findings were made in 2002 in Uganda, who found out that sleeping in a sprayed room reduced the risk of malaria infection by $75 \%$ [15].

IEC materials and malaria case definitions were not seen at the clinic or in the community. Information needs to be available for the community so that the literate ones can read for themselves even if they do not come into contact with a health worker to give them interpersonal health education. This is another way of reducing malaria incidence if the knowledge gained through IEC material translates into behaviour.

The maintenance of minimum stocks of drugs was not being done at the clinic, and no educational strategies to address malaria risk factors had been developed yet. There were still no malaria IEC materials, and mosquito repellents were not being used yet in Binga. 
Mosquito repellents were not stocked in local shops.

\section{Conclusion and Recommendations}

The outbreak mainly affected children under the age of fifteen years. There was poor knowledge on malaria for both cases and controls. The district was inadequately prepared for the outbreak and, as a result, responded late. All the cases were treated in line with the national malaria case management protocols. The late response coupled with a shortage of resources to open satellite clinics had a negative impact on the control of the outbreak. Significant risk factors for contracting malaria in Siansundu were fetching water before sunrise, worshipping outside at night, and having inadequate mosquito nets in the household. Wearing long clothing at night was significantly protective against malaria. The non-spraying of some rooms and shortage of mosquito nets may have contributed to the outbreak. Close monitoring of the spraying programme, allocation of adequate time for net registration and increasing the number of net distribution points; the design of educational strategies which address the risk factors for malaria, provision of malaria IEC materials for Siansundu; stocking of mosquito repellents in local shops and allocation of a Tonga speaking

\section{References}

[1] World Health Organisation; Malaria Factsheet;2013 www.who.int.

[2]Centers for Disease Control and Prevention; Malaria, www.cdc.gov.

[3] World Health Organisation; Malaria, International Travel and Health www. who.int.

[4] World Health Organisation; 2010, Guidelines for the Treatment of Malaria; $2^{\text {nd }}$ Edition, www.who.int.

[5] World Health Organisation, 2010, Centers for Disease Prevention and Control; Technical Guidelines for Integrated Disease Surveillance and Response in the African Region, $2^{\text {nd }}$ Edition. supervisor in all spraying teams; the maintenance of minimum stock levels for antimalarial drugs and the convening of the district epidemic committee meeting at regular intervals could assist in future outbreak prevention and control.

\section{Conflict of Interest}

The authors declare no conflict of interest.

\section{Authors Contributions}

All authors were responsible for the conception of the problem, design, collection, analysis, and interpretation of data, and drafting the final article. The manuscript was read and approved by all authors.

\section{Acknowledgements}

I express sincere gratitude to the University of Zimbabwe (UZ) staff for support and guidance during the conduct of this study. I would like to particularly thank my supervisors, Professor M Tshimanga, Dr. Hwalima, C Sibanda, and Dr. Masuka, for their guidance. I am also thankful to the Health Studies Office (HSO), Centres for Disease Control and Prevention (CDC) and Provincial Health Executive (PHE), Matabeleland North Province, District Health Executive Binga, and the study participants.

[6] World Health Organisation: 10 Facts on Malaria in Africa www.who.int.

[7] Centers for Disease Prevention and Control, 2011, The Global Malaria Epidemic, 2011 www.globalhealth.com.

[8] Ministry of Health and Child Welfare, 2009, Guidelines for Management of Malaria in Zimbabwe [9] Matabeleland North Province, 2012, Malaria Presentation-Malaria Conference.

[10] Matabeleland North Province, 2013 Week 4, Weekly Disease Surveillance Data.

[11] Sibanda C, Gombe N.T and Hazangue P, 2010, Risk Factors for Contracting Malaria in Siansundu, Binga; (Unpublished). 
[12] Ahmed S.M; Haque R and Hossain A, 2009, Knowledge on the Transmission, Prevention and Treatment of Malaria among two Endemic Populations of Bangladesh and their Health-Seeking Behaviour: Malaria Journal, (8)173. https://malariajournal.biomedcentral.com/articles/10 .1186/1475-2875-8-173.

[13]Lairez E; 2005, The intersectoral response to the malaria epidemic in Ethiopia, Humanitarian Exchange Magazine; Issue 31 https://odihpn.org/magazine/the-intersectoralresponse-to-the-malaria-epidemic-in-ethiopia-in2003-an-assessment/.
[14]Basch J.A; Gu J.J; Msellem M; Martenson A and Ali A.S, 2013, Perception of malaria risk in a setting of reduced malaria transmission; Malaria Journal, https://malariajournal.biomedcentral.com/articles/10 .1186/1475-2875-12-75.

[15] Guyatt H.L; Corlett S.A; Robinson T.P; Ochola S.A and Snow R.W, 2002, Malaria prevention in highland Kenya, Indoor residual house spraying versus insecticide-treated bed nets: Tropical Medicine and International Health,7(4):298-303 https://onlinelibrary.wiley.com/doi/pdf/10.1046/j.13 65-3156.2002.00874.x. 